\title{
Neuroplasticity and Antipsychotics in Treatment of Schizophrenia
}

\section{Kamal SM* and Dine SE}

Department of Pharmacology, Faculty of Medicine, Ain Shams University, Cairo, Egypt

*Corresponding author: Sahar Mohamed Kamal, Professor of Pharmacology, Faculty of Medicine, Ain Shams University, Cairo, Egypt, Tel: 20224186992; E-mail: saharkamal2003@hotmail.com

Rec date: January 22, 2015; Acc date: May 25, 2015; Pub date: May 28, 2015

Copyright: (c) 2016 Kamal SM, et al. This is an open-access article distributed under the terms of the Creative Commons Attribution License, which permits unrestricted use, distribution, and reproduction in any medium, provided the original author and source are credited.

\section{Short Communication}

Neuroplasticity is pointed to the ability of the brain to change its molecular and structural characteristics that hinder its function. The main pathophysiological alteration in schizophrenic patients is the occurrence of a major deficit in cognitive process that is under the control of the circuitry of the dorsolateral prefrontal cortex (DLPFC) $[1,2]$. Additionally, this cognitive deficit in schizophrenia is partially related to the marked decrease in dopamine [DA] input to the DLPFC. However and fortunately, a compensatory response in the form of upregulation of D1 receptor in this area of brain results in a great improvement of memory-related DLPFC activity.

Studies performed by Elvevag, Goldberg [3] and Black [4] assumed that schizophrenic patients suffered from brain atrophy, neurotoxicity, or neurodegeneration that involved loss of neurons in the gray matter. Additionally, the reduction in brain volume was due to shrinkage of the neuropil surrounding the neurons with marked reduction in dendrite length that was associated with a reduction in the number and size of dendritic extensions.

This disorder is related to an alteration in the functional activity of the dorsolateral prefrontal cortex (DLPFC). DLPFC is a brain region that is related to the control of executive function. Any abnormalities in the function of DLPFC are strongly related to the development and progress of schizophrenic symptoms [5].

Every dendritic spine of neurons of DLPFC is composed of an excitatory synapse due to the presence of the excitatory amino acid mediator, glutamate. When neurodegenerative changes start in this area of brain, it is also associated with a decrease in neurotropin 3 (NT-3, as a brain growth factor) causing a brain tissue loss during schizophrenia together with a decline in growth factors that are critical in brain development, neuroplasticity, and synaptic connectivity [6,7]. On the other hand, it was proved that the pathological alteration in the density of lamina of each spine played a crucial role in the development of schizophrenia.

No animal study has found any effect of first generation antipsychotics (FGAs) e.g. Haloperidol on neurogenesis or neuroplasticity as proved by [8]. Furthermore, it was found that haloperidol possesses a neurotoxic action on DLPFC by apoptotic cell death with a reduction in neurotropins, such as brain-derived neurotropic factor (BDNF) as brain growth factors. Approximately, no human study was interested to do further investigations by this generation in schizophrenia $[9,10]$.

In contrary to FGAs, many second generation antipsychotics (SGAs) stimulate neurogenesis in the adult rat' brain especially hippocampus and DLPFC $[8,11]$.

Fortunately, in repeated restrained rats with psychotic manifestations, quetiapine, risperidone and Paliperidone, an SGA and the active metabolite of risperidone and is now approved for the treatment of schizophrenia, were found to be able to stimulate neurogenesis in hippocampus and other brain areas related to memory maintenance in these experimental rats $[12,13]$.

Hippocampal neuroplasticity is dependent on neurotropins as nerve growth factor (NGF) and BDNF to help maintenance of memory and cognitive functions in elderly [14,15]. Meanwhile, a marked hyperplasia of the striatum is found to be associated with changes in the morphology and the number of synapses, mainly of the glutamatergic type $[16,17]$. The above findings support the assumption that SGAs also improve neuroplasticity in brain regions whose damage is responsible for the pathogenesis of schizophrenia such as the prefrontal and limbic areas. There is strong evidence that SGAs increase the neuronal growth factor "neurotropin-3" in the cortex of people with schizophrenia. This increase would help neuroplasticity with improvement of mental functions in such patients $[16,18]$. The results of these human studies open the way in front of many researchers in this field to do long-term studies with various SGAs to study the possible beneficial regional neuroplasticity-induced by these drugs.

Similarly, many clinical studies reported, in a confident manner, that neurotropins such as (NGF) and BDNF are decreased in schizophrenic patients of different age groups. These neurotropins have a crucial role in neuroplasticity and protection against apoptosis that is a part of pathogenesis of this disease [19].

Quetiapine actually stimulates BDNF expression and reduces the immobilization that accompany the exposure to stress in rat hippocampus [20] as well as significantly increases BDNF and fibroblast growth factor 2 (FGF-2) mRNA in rat's hippocampus, neocortex either these rats are stressed or not [21-23].

Recently, Gamma-aminobutyric acid (GABA) is thought to play a role in the pathophysiology of schizophrenia. There is a strong evidence that schizophrenic patients will get a lot of therapeutic benefit when treated with drugs that result in an increase in the expression of postsynaptic GABAA receptors. This increase will be considered as a compensatory mechanism to the deficit in GABA release from DLPFC neurons with an increase in the efficacy of GABA neurotransmission at DLPFC. This will be of great therapeutic benefit in schizophrenic patients as drugs acting on GABA receptors would augment the neuroplasticity modifications associated with improving memory with marked improvement of negative symptoms [24]. Studies are focused on the action of both typical and atypical antipsychotics on GABA concentration in basal ganglia and the anterior singulate nucleus of schizophrenic patients. The results were very promising as regards the increase in GABA concentration in these areas of patients measured in a proton magnetic resonance spectroscopy study with its proposed beneficial effects in modification of impaired memory and enhancement of neuroplasticity in such disease. However, more studies 
Citation: Kamal SM, Dine SE (2015) Neuroplasticity and Antipsychotics in Treatment of Schizophrenia. J Neurol Disord 3: 232. doi:

Page 2 of 2

are needed to support the GABAergic therapeutic action of SGAs especially as there is a strategy to develop drugs acting on GABAergic system to treat psychiatric diseases as depression and bipolar disorders $[25,26]$.

A lot of clinical studies summarized the main pathological changes of schizophrenia to be due to dopaminergic overstimulation which can lead to cell death [27], glutamate excitotoxicity [28], GABA dysfunction [29], impaired anti-apoptotic signaling, mitochondrial dysfunction [30], decreased nitric oxide biosynthesis [31] and oxidative stress [32].

Currently, there is a strong recommendation to design many clinical trials, whose aim is to prove in a definite manner the possible improvement of neuroplasticity and the expected reversal of the associated pathological changes that affect the brain of schizophrenic patients by SGAs when used in the treatment of schizophrenic patients. These trials will help a lot to reach curative drugs to this mental disease [17,33-35].
In conclusion, there is strong evidence that atypical antipsychotics possess an important role in neuroplasticity and neuroprotection when administered to schizophrenic patients. The results of many studies pointed to this obvious neuroprotective effect and to the capacity of neurogenesis of SGAs. This will open a new era to investigate new drugs, especially GABAergic drugs, in an attempt to reach a complete cure of chronic neuropsychiatric diseases such as schizophrenia. This target can be achieved if studies can develop new drugs that depend mainly in their mechanism of action on both neuroplasticity and regeneration of abnormal neuronal circuits so they can replenish the brain neurotropic factors. These drugs could successfully be used in treatment of recurrent episodes of schizophrenic patients.

These impressing studies will help to announce very soon in the future the appearance of new new drugs to treat this psychotic illness. Further advances in psychopharmacology may include an array that properly investigates the neuroplastic mechanism of action of these new drugs to reach a complete brain repair that is needed in such destructive mental diseases. 